Portland State University

PDXScholar

$1-1-1995$

\title{
Molecular fluorescence spectroscopy in the vicinity of a microstructure
}

P.T. Leung

Portland State University

Thomas F. George

Follow this and additional works at: https://pdxscholar.library.pdx.edu/phy_fac

Part of the Physics Commons

Let us know how access to this document benefits you.

Citation Details

Leung, P. T. and George, T. F. 1995 " Molecular Fluorescence Spectroscopy in the Vicinity of a Microstructure." J. de Chim. Phys. (France) 92: 226-247.

This Article is brought to you for free and open access. It has been accepted for inclusion in Physics Faculty Publications and Presentations by an authorized administrator of PDXScholar. Please contact us if we can make this document more accessible: pdxscholar@pdx.edu. 


\title{
Molecular fluorescence spectroscopy in the vicinity of a microstructure
}

\author{
PT Leung1, TF George 2*
}

'Department of Physics/Environmental Science and Resources Program, Portland State University, PO Box 751. Portland, Oregon 97207-0751; 2 Departments of Chemistry and Physics, Washington State University, Pullman, Washington 99164-1046, USA

- Correspondence and reprints.

\section{Abstract}

A brief review is presented on fluorescence spectroscopy of molecules in the vicinity of a microstructure, which could be a surface structure, clustered ions/atoms, a colloidal particle in a solution, or a tip in certain molecular probes. This structure can be viewed as microscopic, mesoscopic or macroscopic, depending on the specific environment in which the molecule is located. An overview is given for selected experimental and theoretical aspects of this phenomenon, emphasizing some of the previous modeling work of the authors. Discussion of possible future attempts to explore the phenomenon in more detail is presented at the end.

\section{Resume}

Le sujet de cet article est la fluorescence des molécules au voisinage d'une microstructure telle qu'une surface, un agrégat ionique ou atomique, une particule colloidale en solution ou un embout de montage expérimental. En fonction de l'environnement cette structure peut être considérée comme microscopique, mesoscopique ou macroscopique. On passe en revue un certain nombre des aspects expérimentaux et théoriques du problème, l'accent étant mis sur les modèles proposés précedemment par les auteurs. On discute, enfin, des tentatives d'une étude plus détaillée de ces phénomènes.

Keywords: fluorescence, molecular decay rates, frequency shifts, microscopic/ mesoscopic environment

Introduction

Ever since the pioneering work of Purcell in 1946 (1] which proposed the possibility of shortening the NMR relaxation time due to interaction with a "resonator" in the vicinity of the system (nuclei), it has been recognized that the absorption and emission spectroscopy of any microscoplc (molecular) system could be dramatically modified via interaction between the system and its environment. Additional energy transfer channels are opened up due to this interaction, ultimately leading to modification of the absorption crosssection, the decay rate and the level position of the molecular species. There has been considerable interest in studying this problem in the last two decades, covering a wide varfety of situations ranging from studies in fundamental quantum electrodynamics (QED) to surface physics. Some examples 
are: fluorescence at interfaces $[2,3]$, cavity QED [4], solvated molecules in clustered lons/atoms [5] as well as in colloidal solution [6] and, more recently, single-molecule detection (SMD) via near-field optics [7]. The purpose of these kinds of studies is generally twofold : (1) to understand how the absorption/emission properties of the "admolecules" could be modified to achieve certain desired photophysical or photochemical processes, and (2) to understand the structure/properties of the "environment" via monitoring the modified optical properties of the embedded molecule. While the effects due to a "macroscopic environment" such as a surface or cavity depend mainly on the dielectric (optical) properties and geometry of the environment, those owing to a certain "microscopic/mesoscropic environment" such as clustered lons/molecules may depend very much on the details of the molecular structure of the systems.

In this review, we concentrate on the fluorescence of molecules such as dyes in the vicinity of a "microstructure" .. a generic term adopted here to include any of the aforementioned macro- or microscopic environments such as an "island" site on a rough surface, a colloidal particle or clustered ion in solution, or a mesoscopic cavity in which the fluorescent molecule is confined. It is thus appropriate to first review the experimental situation in order to motivate our following theoretical discussion to understand and simulate.this phenomenon.

\section{Review of Experiments}

In this section, we give a brief summary of various experimental observations which are relevant to the spectroscopic properties of molecules in the vicinity of a microstructure.

\section{(a) Rough surfaces:}

While the study of the spectroscopy of molecules adsorbed at interfaces has interested surface scientists for quite some time, the recent surge of activities in this area occurred after the discovery of the dramatic millionfold enhancement of the Raman scattering signal from organic molecules adsorbed on noble metal surfaces (electrodes) [8]. Soon after, it was recognized that one main cause of this enhancement is due to the huge localized surface electromagnetic (EM) field originating from resonant excitation of the surface plasmon in the presence of surface roughness [9]. This then opened up the study of possible enhancement of other types of surface spectroscopy (e.g. fluorescence) and photoprocesses (e.g. photochemistry) on rough metallic surfaces $[3,10]$.

For some time, it was belleved that these other processes are much less likely to get enhanced due to the very serlous damping of the excited molecular states via nonradiative energy transfer to the substrate when the molecule is close to the surface [2]. In photochemical processes such as 
photodissociation, this surface-damping effect will at least manifest itself as a competing factor to weaken the effect from the enhanced local aM field $(10,11]$. In the case of fluorescence, it was thought that most of the emission will be quenched when the molecule is very close to the surface, and in addition, a preliminary theoretical analysis seemed to imply that the presence of surface roughness will only further facilitate the induced damping to quench the fluorescence [12]. However, more comprehensive analysis later showed that this may not be necessarily the case (13). In particular, by modeling the roughness as a collection of islands [14] or as a grating, it was

demonstrated that, depending on various factors such as the surface morphology, orientation, emission frequency and other properties of the admolecule, roughness can in some cases lead to suppression of the induced decay $(13,15]$ !

In a recent experiment by Ahmadi and Rusling [16], enhanced fluorescence was indeed observed from pyrene adsorbed at a rough silver electrode. In this experiment, the Ag electrodes were dipped into a pyrene-dimethylformamide solution, and fluorescence was observed from thin-layer electrochemical cells. It was found that the quantum yield depended on the potential across the electrodes due to the fact that pyrene has a very low adsorption coefficient for silver, and that there could be structural reorganization of surfactant aggregates on the electrode surface. In addition, a rough $\mathrm{Ag}$-foil electrode was found to enhance the emission from pyrene as compared to electrodes of. vapor-deposited $\mathrm{Ag}$ films in aqueous micellar media. We have since been encouraged by this indirect support for our modeling results $[13,15]$ and have set forth to improve our models by incorporating quantum effects as discussed shortly in subsection (c).

In order to compare more closely with varlous modeling results and to achieve further improvements, Ahnadi and Rusling proposed to quantify the absolute concentration of the surfactants and to prepare their electrode roughness in a reproducible fashion. On the other hand, it may even be more feasible to perform the fluorescence experiments on an "island surface." since it has been reported recently that such a surface could be prepared with spherical islands of controllable sizes [17]. In this case, the effects of the distribution of the islands could also be modeled by means of certain twodimensional effective-medium or fractal-cluster theories [18] similar to the three-dimensional treatment recently reported for the case of a substrate of composite materials [19].

\section{(b) Clustered atoms:}

Solvation effects on the spectroscopy properties of dissolved molecules have been studied for a long time. Some examples from more recent works include the study of electronic oscillator strengths for solvated $\beta$-carotene and pyrazine molecules [20] and the red shifts in electronic spectra of certain aromatic molecules bound to rare-gas solvent atoms [21]. In addition, these modified oscillator strengths, spectral shifts and radiative lifetimes were 
also studied for large molecules such as 9,10-dichloranthracene (DCA) and retracene and their complexes embedded in clusters of inert gas atoms (5). In this latcer study, it was possible to examine the modified effects as a function of the cluster size, and thus the approach to the limit of a bulk solvent could be monitored. We give a brief review of these works below: The 9,10-DCA system was studied since it has an emission quantum yield very close to unity from the origin of $s_{1}$. This implies that the measurement of the lifetimes gives results directly for pure radiative lifetimes. This is true even in the presence of the host cluster, since all of the inert gases studied have essentially real dielectric functions and are thus nondissipative for the emission from the organic molecule. Different nozzles were used to synthesize the 9,10-DCA clusters with different inert gases including Ar, Kr and $X e$, along with different experiments for measuring absorption, quantum yield and lifetimes $[5,22]$. A pulsed dye layer was used to cross excite the cluster beam, and a time-resolved study was performed for the fluorescent emission from the clusters. The size of the clusters was controlled by monitoring the nozzle backing pressure of the inert gas. Among other results, it was found that the fluorescent lifetime of 9,10-DCA is greater than both that of a free molecule and that of the molecule in bulk solution: it increases as the cluster size begins to grow, then reverses its trend until it saturates at a value which is still $12 x$ greater than the free molecule value $(-23.3 \mathrm{~ns})$. Furthermore, the red shift in the absorption spectra was observed also to increase and then saturate at about the value for the bulk solution case. It was also observed that both the lifetime and frequency shift correlate with the polarizability of the inert gas.

While there exists quantum perturbative theory $[20,23]$ as well as microscopic generalizations of the classical Onsager model [21] to account for the above spectroscopic observations for a fluorescent molecule in a cluster, it was shown that the simplest (yet adequate) theoretical description for such phenomena could be based purely on classical electromagnetic theory [22]. It was further shown that, as long as the interaction among the solvent molecules (the clusters) is negligible, this simple electromagnetic theory is equivalent to the quantum theory [24]. In this approach, one can classify the problem according to four regimes of cluster size: (1) microscopic regime up to a few tens of the solvent molecules in which one needs to account for the structural detalls of the cluster; (ii) electrostatic regime up to sizes of about $10 \mathrm{x}$ of the emission wavelength $\lambda$; (iii) electromagnetic regime (size - $\lambda$ ); and (iv) bulk regime with sizes much greater than $\lambda$. It is partly due to these previous findings in modeling fluorescent spectroscopy from an organic molecule in a cluster that motivate us to discuss a fully electrodynamic theory of dipoleparticle interactions in the following section on Theoretical Modeling. 


\section{(c) Solutions with collotdal/suspension particles and other fractal} substrates:

The spectroscopy of molecules near colloidal or fractal substrates has been of great interest in both experimental and modeling work since the discovery of surface-enhanced Raman scattering (SERS). For example, there have been studies of electronic energy transfer between adsorbate pairs such as rhodamine 6G-malachite green on porous silicas which show the fractal nature of the substrate surfaces [25]. More recently, photochemical decomposition of organic molecules were studied on colloidal silver surfaces, where it was found that the efficlency of the photoprocess is enhanced only in the presence of the colloids and is very dependent on the orientation of the adsorbed molecules [26]. The same kinds of electromagnetic effects are expected in these fractal/colloidal systems as they occur at roughened surfaces, which are one of the main reasons for the millionfold enhanced SERS signal [9]. In fact, recent studies of the optical properties of fractal clusters [27] and their applications to modeling SERS [28] have indeed revealed huge electromagnetic enhancement factors from these surface morphologies.

Aside from modeling SERS, colloidal systems are interesting in their own right as providing an interesting medium with which external molecules can interact. Furthermore, certain colloids such as gold also show fractalclustering structure and have been studied using other imaging methods [29]. Thus it would be of interest to look for novel spectroscopic phenomena from molecules near or embedded in such a colloid. Indeed, among others, a group at Brown University has recently studied this phenomenon with fluorescing dye molecules embedded in a collofdal suspension of polystyrene spheres [30] and has observed very interesting behavior concerning the lifetimes (Inversed decay rate) of the dyes. In particular, they found that at high densities of the colloidal particles, the lifetimes are lengthened whereas they are shortened at low densities of the polystyrene particles. More interestingly, they found lately that the coherent optical scattering from the colloidal particles (which helps explain the above observation on lifetimes) could be used to generate laser light with a relatively low threshold excitation energy [31]. Using a suspension of titanium dioxide particles in methanol which contains the dissolved dye molecules, the Brown group has been able to make the dye lase at $617 \mathrm{~nm}$.

All these serve as illustrations of the very rich phenomenon of fluorescence from molecules in an environment with clustered particles or irregular (fractal) geometry. To a certain extent, it was also found that the phenomenological description modeling the molecule as an oscillating dipole can account for most of these observations [30]. Recently, we have also extended this modeling to composite substrates with particulates distributed through a host such as cermet [19]. Our model accounts for both randomized and fractalclustered distributions of these particulates and should be capable of modeling the experiments described above. 


\section{(d) Single-molecule detection vil near-field optics:}

Another very exciting area recently developed which has high relevance in our present context is the fluorescence imaging of molecules using the nearfield optics technique [7a]. Here a spot of light (introduced by a fibre) smaller than the optical wavelength is scanned in close proximity to the sample supported on a substrate. Resolution is achlevable down to the sub-diffraction iimit, and single-molecule detection is possible. This technique has been successfully applied to detect a large variety of single molecules including dyes and proteins. The imaging is based on the fluorescence from the sample molecule in the vicinity of the probe. Recent time-resolved experiments have reported signiflcant modification of lifetimes of molecules being probed by the tip of the near-field optical microscope $[7 b]$. Hence, the modeling and understanding of the modification of fluorescent properties of these molecules in the vicinity of the probe are extremely crucial to the analysis of the image obtained from such an approach.

\section{Theoretical Modeling}

While exact modeling has to be ultimately quantum mechanical (QM) - in the sense that the environment-induced molecular properties are calculated from QM perturbation theory for transition probabilities with a $Q M$ many-body description for the electronic wave function of the environment - a simpler and less computational appoach is a phenomenological theory which we shall deal with mainly in this section. This latter approach models the fluorescing molecule as a radiating dipole, and environment-induced effects are accounced for by solving the "Sommerfeld-type" problem (1.e. the modified electrodynamics) [32] taking into account the geometrical boundary as well as the dielectric (optical) properties of the environment (i.e. the "microstructure"). We shall show below that, while this approach is mainiy classical in Its nature, certain important $Q M$ effects can indeed be introduced in a phenomenological and economical way. Thus, following this approach, the molecular transition dipole $(\vec{\mu})$ between the fluorescing states is governed by the equation of motion for a damped harmonic oscillator:

$$
\ddot{\vec{\mu}}+\gamma_{0} \dot{\vec{\mu}}+\omega_{0}^{2} \vec{\mu}-\frac{e^{2}}{\mathbf{m}} \vec{E}_{R}
$$

where $\mu_{0}$ and $\gamma_{0}$ refer to the intrinsic transition frequency and level width (decay rate) for an isolated (free) molecule, and the dipole is driven by the field $\left(E_{R}\right)$ reflected from the boundary of the microstructure, thus providing a mechanism for the Induced effects on the molecular properties. Approxinate solutions can then be sought which finally express the modified decay rate and frequency (level) shift normalized to $\gamma_{0}$ as [2] 


$$
\begin{aligned}
& \frac{\gamma}{\gamma_{0}}-1+\frac{3 q}{2 \mu_{0} \mathrm{k}^{3}} \operatorname{Im}\left(E_{0}\right), \\
& \frac{\Delta \omega}{\gamma_{0}}=-\frac{3 q}{4 \mu_{0} \mathrm{k}^{3}} \operatorname{Re}\left(E_{0}\right),
\end{aligned}
$$

with $\mu_{0}$ and $E_{0}$ being the magnitudes of $\mu$ and $E_{R}$, respectively, and $k$ the wave number. As is clear from Eqs. (1) and (2), the model depends on phenomenological input of the total quantum yield (q) of the fluorescing molecule as well as the computation of $E_{R}$, which in turn depends strongly on many factors including: molecular orientation with respect to the microstructure boundary, emission frequency $(\omega)$, distance (d) between the molecule and the boundary, and geometrical and optical properties of the environment. Being as simple as it is, this approach was found to model quite adequately the fluorescence of admolecules at surfaces in the 70's and 80 's [2], although the experimental situation must have a relatively large moleculesurface separation $d\left(-10^{3} \mathrm{~A}\right)$, and the molecular coverage must be sufficiently low such that intermolecular interations can be neglected [33]. Given these Iimitations, the main task in this modeling then involves the calculation of $E_{R}$ for a specific environmental structure. In the following, we shall simplify this structure by referring largely to a typical dielectric sphere of unlimited diameter which could be microscopic $(2 a<10 \AA)$, mesoscopic $(10 \AA<2 a<10 \mathrm{~mm})$ or macroscopic $(0.01 \mu \mathrm{m}<2 \mathrm{a})$. In solving the electrodynamics problem involving the fluorescing dipole and the structure, we shall pay special attention to the importance of the dynamic and nonlocal responses of the structure to the molecular radiation, and hence we shall divide our review of the various calculation of $E_{R}$ in the literature into the following three subsections:

\section{(a) Electrostatics approach:}

This simplified solution to the problem was adopted by many people in the 70 's and early $80^{\prime} \mathrm{s}$ due to the tremendous gain in mathematical simplification using an "Image theory" (IT) treatment. The interaction between the molecule and its environment is completely described by that between the molecule and a "fictitous image" residing in the structure. Structures of different geometrical boundaries were treated in this way including the case of a flat surface substrate [34], a rough surface [12] and a spherical island substrate [35]. This simple theory did have certain success (e.g. it correctly predicts the $d^{-4}$ dependence for $\gamma$ and $\Delta \omega$ ), and it was believed to be accurate enough for many experimental situations since the emission wavelength $\left(-10^{3} \AA\right.$ in the optical region) must usually be much greater than $d\left(-10^{2} A\right)$ for appreciable structure-induced effects to be observed. Thus within IT, for example, $\gamma$ for a molecule outside a spherical particle can then be given by the expressions (for simplicity, all the modeling here will assume $q-1$ ) 


$$
\begin{aligned}
& \frac{\gamma_{\perp}}{\gamma_{0}}-1+\frac{3}{2 k^{3}} \sum_{n=1}^{\infty}\left(I m \alpha_{n}(\omega) \frac{(n+1)^{2}}{d^{2 \cdot(n+2)}}\right), \\
& \frac{\gamma_{\|}}{\gamma_{0}}-1+\frac{3}{2 k^{3}} \sum_{n=1}^{\infty}\left[I_{n}(\omega) \frac{n(n+1)}{2 d^{2(n+2)}}\right),
\end{aligned}
$$

where $\perp$ and $\|$ denote the two orthogonal orientations (radial and tangential) of the molecular dipole with repect to the particle, and $a_{n}$ the $n$-th polarizability of the sphere. As is clear thus far, the dielectric properties of the structure are completely accounted for by a local dielectric function $\epsilon(\omega)$ which is dependent only on the frequency, not on the wave vector of the molecular emission. We shall explore the limitation of this description later in subsection (c) below. However, even granted this local dielectric response from the environment, the static theory could be quite inaccurate in certain realistic situations as illustrated in the following.

\section{(b) Dynamical approach:}

Although the electrodynamical solution is available for flat boundaries ever since the first comprehensive treatment of the problem of surface-modified fluorescence using the phenomenological approach [2], for the case of rough or spherical boundaries the solution was less often available due to the mathematical complexity in matching the boundary conditions when solving the electrodynamics problem. Instead, static solutions have been often resorted to modeling the spectroscopic properties of molecules in the vicinity of microstructures $[12,14,35]$. In 1982, Ruppin published the dynamic solution for an admolecule in the vicinity of a spherical particle using the conventional vector harmonics expressions for the reflected field $E_{R}[36]$. Soon after, it was recognized that the close distance requirement $(d \ll \lambda$ ) is not sufficient for the static approximation to be accurate. In particular, the image theory could fail appreciably under the following two situations: (i) when the environment is highly conducting so that the skin depth at the wavelength of the molecular emission is much smaller than $d ;$ and (ii) in the presence of a conducting microstructure where nonradiative surface plasmon excitation in the structure could be coupled back into the radiative modes such that "resonant radiative transfer" takes place (at a certain emission frequency) between the excited molecule and the structure, where the static theory simply cannot account for any radiative transfer phenomenon $[37,38]$. Figures 1 and 2 illustrate these two issues clearly.

Hence it is desirable to have a fully dynamical theory (such as that of Ruppin) modeling the interaction between an admolecule and a microstructure. In 1987, Chew extended Ruppin's theory to allow for the molecule to reside within the spherical particle [39] - with linitation only to the case of nondissipative particles - thus doing away with any nonradiative transfer between 


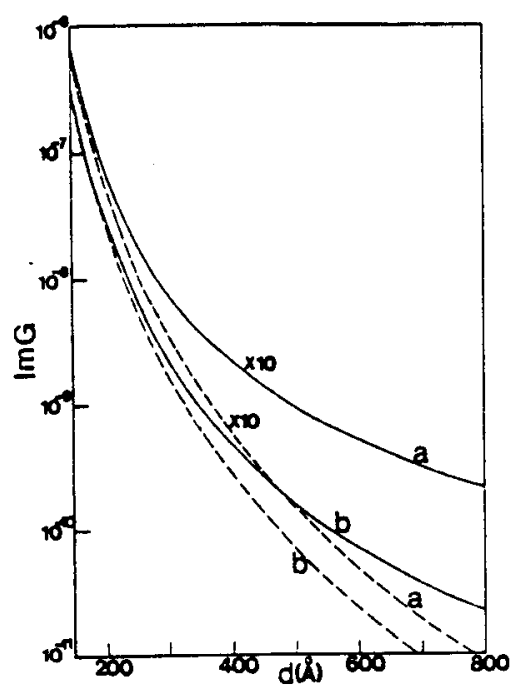

Fig. 1. - Comparison between the image theory (IT, dashed curves) and dynamical theory (solid curves) where ImG (G- $\mathrm{E}_{0} / \mu_{0}$. Eq. (2)) is plotted vs $d$ for a (a) $A G$ and (b) Ni sphere. The failure of IT for the highly-conducting Ag (case (a)) is obvious. (Ref. [37])

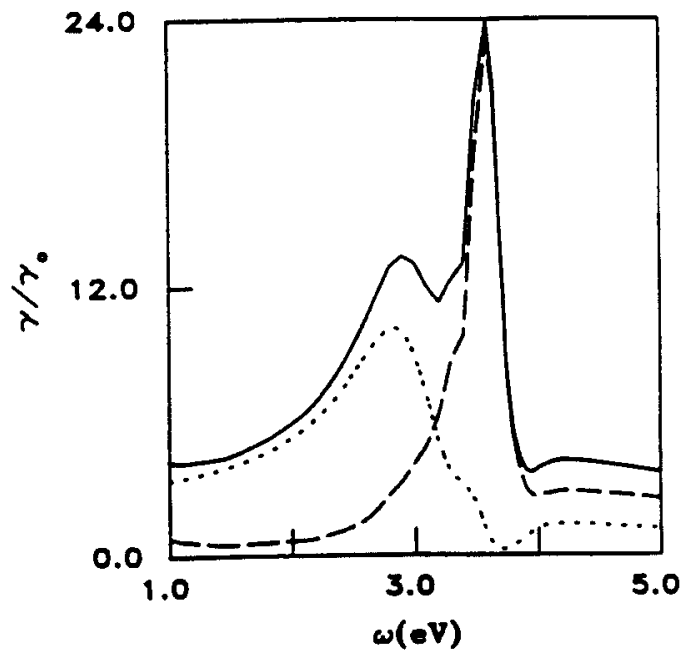

Fig. 2. - Comparison of the decay rate spectrum for IT (dashed curves) and the dynanical theory (solid curves) where "resonant radiative transfer" only shows up in the dynaical theory. The dotted curve shows the radiative contribution. (Ref. $[38]$ ) 
the molecule and the structure. Shortly thereafter, wo set forth to work out a complete treatment to allow for the molecule to lie both outside and inside the particle, and to include the case where the particle can have dissipative dielectric properties [15]. Furthermore, we adopted a slightly different mathematical formalism in solving the electrodynamics of the problem by using the Hertz vector approach, which is parallel to the original theory for flat boundaries [2] and slightly simpler than the approach using vector harmonics in the previous works $[36,39]$. We shall give below a summary of this theory. We shall see that the simplicity of this approach is due to the fact that the expansions of the Hertz functions are more of a scalar type, and one can deduce easily from the Green's function property a simple transformation which relates the fields for the outside-molecule case to those where the molecule is located inside the sphere. Hence it is not necessary to solve again the boundary-value problem for the inside-molecule case [39]. The Hertz vector (II) solution for the dipole-sphere problem was provided by van der Pol and Bremmer $\{40\}$, and also by Fock [41]. For a dipole (of unit dipole moment) located at $(d, 0,0)$ outside a sphere of radius a with the outside (inside) medium characterized by some local dielectric function $\epsilon_{1}\left(\epsilon_{2}\right)$, the Hertz vectors and the nonvanishing field components can be obtained in terms of the spherical Bessel functions $J_{n}$ and $h_{n}^{(1)}$ and the standard "Mie coefficients" $B_{n}$ by solving the appropriate boundary-value problem.

Adopting this formulation and making use of the well-known properties of Green's functions, one can easily obtain via a simple transformation $T$ the solution for the fields in the case where the dipole is located inside the sphere without having to solve again the boundary-value problem as was done prevlously [39]. If we define $T$ by

$$
T\left(\begin{array}{c}
j_{n} \\
h_{n}^{(1)} \\
k_{1}, \epsilon_{1}, \ldots \\
k_{2}, \epsilon_{2}, \ldots
\end{array}\right)=\left(\begin{array}{c}
h_{n}^{(1)} \\
j_{n} \\
k_{2}, \epsilon_{2}, \ldots \\
k_{1}, \epsilon_{1}, \ldots
\end{array}\right)
$$

then it is not difficult to check that the "inside solutions" are easily obtained from the outside solutions through the operation with $T$. To apply the above results for the reflected fields to study the environment-modified spectroscopic properties for the adnolecule via Eq. (2), we have tentatively divided our modeling into three cases of interest below. Here we just give a brief survey of the results, where more detalls can be found in Ref. [15]:

\section{Case (i): "Surface" problem}

Here the molecule is located (say, In vacuun so that $\epsilon_{1}-1$ ) outside the structure which is modeled as a sphere with dielectric constant given by $\epsilon_{2}=\epsilon^{\prime}+1 \epsilon^{\prime \prime}$, and the "free decay rate" (1.e., in the absence of the structure) 
is given by $2 e^{2} k_{1}{ }^{3} / 3 \mathrm{mw}$, with the symbols carrying obvious meaning. This modeling can, for example, simulate situations for an admolecule in the vicinity of a "surface island" for a rough substrate surface, or of a "microprobe" such as in the case of the near-field optical microscope [7]. The structure in this case can also simulate a colloldal particle or clustered atoms. In addition to calculating the total decay rate as given in Eq. (2), one can obtain the radiative rate as

$$
\gamma^{R}-\frac{1}{W} \int_{(r+\infty)} d n r^{2} \vec{s} \cdot \dot{n},
$$

with $\vec{S}$ being the Poynting vector and $W$ the average energy of the dipole [15] The nonradiative part can then be obtained by the difference between the total and the radiative values. Using Eqs. (2) and (5), one finally obtains for the radial dipole [15]

$$
\begin{aligned}
& \frac{\gamma_{\perp}}{\gamma_{0}}-1+\frac{3}{2} \operatorname{Re} \sum_{n=1}^{\infty}(2 n+1) n(n+1) B_{n}\left[\frac{h_{n}^{(1)}\left(y_{1}\right)}{y_{1}}\right]^{2}, \\
& \frac{\gamma_{1}^{R}}{\gamma_{0}}-\frac{3}{2} \sum_{n=1}^{\infty} n(n+1)(2 n+1)\left[\frac{j_{n}\left(y_{1}\right)+B_{n} h_{n}^{(1)}\left(y_{1}\right)}{y_{1}}\right]^{2},
\end{aligned}
$$

where we have $y_{1}-k_{1} d$ and $k_{1}-\sqrt{\epsilon_{1} \omega / c}$, with similar expressions for $\gamma$ and $\gamma_{i}^{R}$ for the tangential dipole. The induced frequency shifts can also be obtained via Eq. (2) as

$$
\frac{\Delta \omega_{1}}{\gamma_{0}}=-\frac{3}{4} \operatorname{Im} \sum_{n=1}^{\infty}(2 n+1) n(n+1) B_{n}\left[\frac{h_{n}^{(1)}\left(y_{1}\right)}{y_{1}}\right]^{2} .
$$

\section{Case (ii): "Cavicy" problem}

Hexe the molecule is located inside a spherical vacuum $\left(\epsilon_{2}-1\right)$ with a dielectric $\left(\epsilon_{1}-\epsilon^{\prime}+\epsilon^{\prime \prime}\right)$ occupying the whole space outside the vacuum. The total decay rates can readily be obtained from the outside solutions through the transformation T (Eq. (4)) as discussed above, where we obtain for the radial dipole

$$
\frac{\gamma_{1}}{\gamma_{0}}=1+\frac{3}{2} R \cdot \sum_{n=1}^{\infty}(2 n+1) n(n+1) E_{n}\left[\frac{j_{n}\left(y_{2}\right)}{y_{2}}\right]^{2} \text {, }
$$


with $E_{n}-T\left(B_{n}\right)$, and similarly for the tangential dipole. More details on the Interesting features of this case, such as in the limlt where the outside medium becomes perfectly conducting, as well as the limitation of this classical description, can be found in Ref. [42]. Again, the Induced molecular frequency shifts can be obtained accordingly. The ultimate quantum treatment of this problem has opened up a new field - cavity quantum electrodynamics which has been actively studied during the past decade $[4,42]$.

Case (iil): "Aerosol" problem

Here we model the molecule as embedded inside a dielectric sphere $\left(\epsilon_{2}-\epsilon^{\prime}+i \epsilon^{\prime \prime}\right)$ with the outside being vacuum $\left(\epsilon_{1}-1\right)$. Again, this "aerosol" could be some real mesoscopic dielectric particle (e.g. glycerol (43]) or clustered atoms. Recent interest in the possibility of lasing and singlemolecule detection for a molecule trapped inside such a particle should be appropriate for modeling in this case [44]. We shall further divide this into two cases for discussion:

\section{(1) Transparent dielectric}

Here the dielectric function of the particle is real and has been treated in the literature using the vector spherfcal harmoics approach. In our approach, we simply make use of the transformation $T$ in Eq. (4) and obtain the total decay rates as in Eq. (8). The radiative rates can be obtained accordingly through $T$ and Eq. (5) [15] for the radial dipole as

$$
\frac{\gamma_{\perp}^{R}}{\gamma_{0}}-\frac{3}{2} \epsilon \frac{3 / 2}{2} \sum_{n=1}^{\infty} n(n+1)(2 n+1)\left[\frac{j_{n}\left(y_{2}\right)}{\rho_{2} \gamma_{2} D_{n}}\right]^{2} \text {, }
$$

where $D_{n}$ is one of the Mie coefficients [15]. (The case for the tangential dipole is similar.) Here there is no nonradiative transfer, and the total and radiative rates can be shown to be identical [39]. The frequency shifts can be obtained similarly.

\section{(2) Absorptive dielectric}

The difficulty of treating this case lies in the calculation of $\gamma_{0}$ in the above equations. This decay rate in the absence of the particle boundary must obviously be nonradiative in nature [15]. We have followed the approach of Agranovich and Dubovskii (AD) [45] to obtain

$$
\frac{\gamma_{0}}{\gamma_{s p}}-\frac{3 z}{64 \pi^{4}} \in " \mathrm{~N}_{0} \lambda^{3} \text {. }
$$


where $r_{s p}$ is the spontaneous decay of the free molecule, $N_{0}$ is the molecular number density of the dielectric medium, $\lambda$ is the emission wavelength, and $z$ is a numerical factor $(-10)$ depending on the lattice structure of the medium. The above holds as long as $\lambda$ is much greater than the lattice constant of the medium and spatial dispersion can be neglected [45]. Using Eqs. (2), (4) and (10), we finally obtain for the radial dipole

$$
\frac{\gamma_{\perp}}{\gamma_{0}}-1+\frac{3}{2} f(\omega) R e\left[\sqrt{\epsilon_{2}} \sum_{n=1}^{\infty}(2 n+1) n(n+1) E_{n}\left[\frac{j_{n}\left(y_{2}\right)}{y_{2}}\right]^{2}\right) \text {. }
$$

with $f(\omega)=\gamma_{s p} / \gamma_{0}$ being the reciprocal of Eq. (10). A similar expression is found for the tangential dipole. The radiative rates can analogously be obtained from Eqs. (5) and ( 9 ) by multiplying each of them by $f(w)$ [15] .

\section{(c) Nonlocal effects:}

While the above phenomenological theory based on classical electrodynamics has been quite successful in explaining certain experimental results [2], it certainly has ifmitations in omitting all the quantum features of the problem. In addition, it is known that this approach only works for low concentrations of both the fluorescent molecules and the surrounding particles, and for relatively large distances between the molecule and the particle boundary (33). Strictly speaking, both the molecule and the particle are quantum mechanical in nature. Even though the molecular transition dipole can be modeled as an oscillating point dipole in many cases, at very close distances ( $\leq 10 \mathrm{~nm}$ ) the wave nature of the surface (particle boundary) will manifest itself so that the above treatment, assuming a sharp boundary, will fail. In fact, the above theory predicts divergent results for both the decay rates and frequency shifts as $d \rightarrow 0$, which is unphysical. The key lies in the assumption of a local dielectric response of the particle which is valid only in regions where the electromagnetic fields vary smoothly throughout [46]. This is not the case when being close to surface. Hence we would expect that one of the most significant modifications to the above theory at close distances will be the nonlocal response of the particle, among other quantum effects. In some previous works, we have explored the possibility of introducing these nonlocal (NL) corrections into the theory in the most economical (phenomenological) way, which we summarize briefly below.

Heving been clear that these $\mathrm{NL}$ effects are only important for very close distances, and with the clarification in subsection (b) above that at such distances the static theory will be accurate enough for most substrates, we go back to the simplex static image theory to introduce the NL effects. In this approach, we see that the results are totally governed by the multipolar polarizability as can be seen fron Eq. (3). Thus we can completely account for the NL effects in this linit if we know how to calculate $\alpha_{n}$ assuming a NL 
dielectric response of the particle. To this end, we refer to the model published by Fuchs and Claro [47].

In the static theory using a local dielectric function for the particle, the usual boundary conditions for the continuity of both the normal component of the displacement vector $\left(D_{\perp}\right)$ and the tangential component of the electric field $\left(E_{\|}\right)$lead to a discontinuity of $E$ across the boundary. To "smoothen" the E-field, Fuchs and Claro introduce a fictitious surface charge at the particle boundary which then makes $D_{\perp}$ discontinuous. By manipulating this latter additional boundary condition. E can then be made to continue across the boundary, which in effect smears out the boundary, in order to account for the quantum (wave) nature of the surface electrons. In dolng this, they were able to obtain a phenomenological expression for the NL multipolar polarizability as

$$
\alpha_{n}(\omega)=\frac{n\left(\zeta_{n}-1\right)}{n\left(\zeta_{n}+1\right)+1} a^{2 n+1}
$$

with

$$
\zeta_{n}(\omega)=\left[\frac{2}{\pi}(2 n+1) a \int_{0}^{\infty} d k \frac{j_{n}^{2}(k a)}{\epsilon(\vec{k}, \omega)}\right]^{-1},
$$

where the specific nonlocal response $c(\vec{k}, \omega)$ is an input taken from microscopic many-body theories [48]. In particular, for a metallic spherical particle, the lowest order correction to the local Drude function $\epsilon(\omega)$ can be determined by a hydrodynamic model [48] and is given by

$$
\epsilon(\vec{k}, \omega)=1-\frac{\omega_{\rho}^{2}}{\omega(\omega+i \Gamma)-\beta^{2} k^{2}} .
$$

With Eq. (14), it turns out that Eq. (13) can be integrated exactly by employing the special properties of the spherical Bessel functions to obtain

$$
\zeta_{n}(\omega)-\left[\frac{1}{c}+(2 n+1)\left[\frac{a \omega_{\rho}}{\beta u}\right]^{2} I_{n+\frac{1}{2}}(u) x_{n+h_{2}}(u)\right]^{-1} .
$$

For other more accurate microscopic models such as the Lindhart-Mermin [48] or modified Lindhart-Merain [49] models, only numerical integration can be performed to study the NL effects in Eqs. (12) and (13). The NL effects on the surface-Induced decay rates and frequency shifts can then be studied via Eq. (2). 


\section{Numerical Illustrations}

To 1llustrate the varfous theoretical results worked out in the last section, we reproduce here some of our previous numerical results. Figure 3.5 show some modeling results for a molecular dipole located in the vicintty of a "surface island," a cavity, and inside a dielectric particle, respectively. Similar results were obtained in Ref. [22] using a slightly different formalism. Figure 6 illustrates some results obtained within our model for a dissipative particle as described in part (2) under case (iii) in the above subsection (b). Note that in all these modelings we have treated one of the two media as a vacuum (e - 1) for simplicity, with the other medium taken as glycerol ( 6 - 2.16) [43] for the case with a real dielectric, and as silver at $\lambda=413 \mathrm{~nm}(6--4.42+0.73 \mathrm{i})[50]$ for the case of a dissipative dielectric. Among other interesting features, one can see, for example, the very different behaviors for the radiative rates at close distances for the two dipole orientations from Fig. 3, the oscillatory behavior from Figs. 4 and 5 , and most interestingly, the possibility of both enhancing and suppressing the decay rates in these two cases. This is consistent with experimental studies in cavity QED as well as fluorescence from molecules embedded in particles. More detailed comments on the modeling results can be found in Ref. [15].

As commented before, the image theory cannot account for any radiative transfer between the molecule and the environment. Nevertheless, a very interesting and simple model has been devised [35] to account for this transfer within the static image theory. We have comparatively studied this model with the results from the exact dynamical treatment in the last section [51]. Figures 7.9 11lustrate results from which one can conclude that in order for the simple model for radiative rates to be valfd, we must require, in addition to the conditions that the radius of the particle (a) and the molecule-boundary distance (d) must be both much smaller than the wavelength $(\lambda)$, that the value of a must not be too large for a fixed d.

Moreover, the results displayed so far are limited to local dielectric response from the particle. The results for nonlocal (NL) response as discussed in subsection (c) above are 1llustrated in Figs. 10 and 11 for both the decay rates and frequency shifts in atomic units, so that some of the results could be compared from those obtained previously using a microscopic theory based on density functional theory [52]. Among other features, one can observe that NL effects (1) are important for close molecule-surface distances, (2) lead to blue-shifted multipolar resonances and anomalous dispersion in the frequency spectrum for decay rates and frequency shifts, respectively, and (3) lead to possible dramatic suppression of surface effects at very close distances from the surface. The comparisons with the microscopic theory for $\gamma$ show completely opposite results. However, there seems to exist very recent experimental data which support the qualitative trends as predicted in our present modeling $[53,54]$. More detafls on this issue can be found in our work 


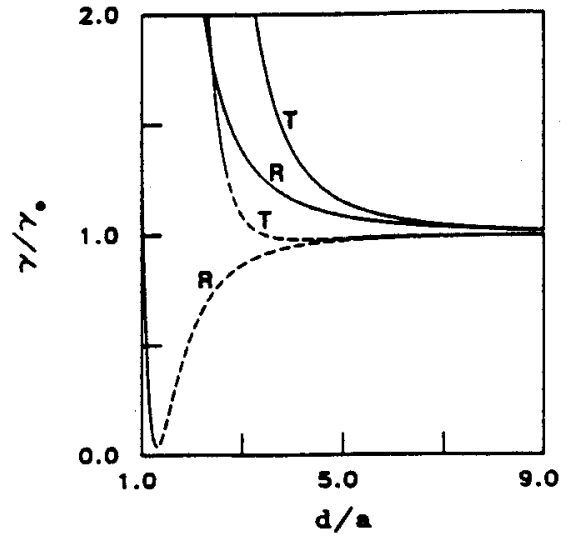

Fig. 3 - Decay rates for a molecular dipole outside a surface island (spherical). The solid (dashed) curves are for radial and tangential dipoles, while $T$ and $R$ denote total and radiative rates, respectively. (Ref. [15])

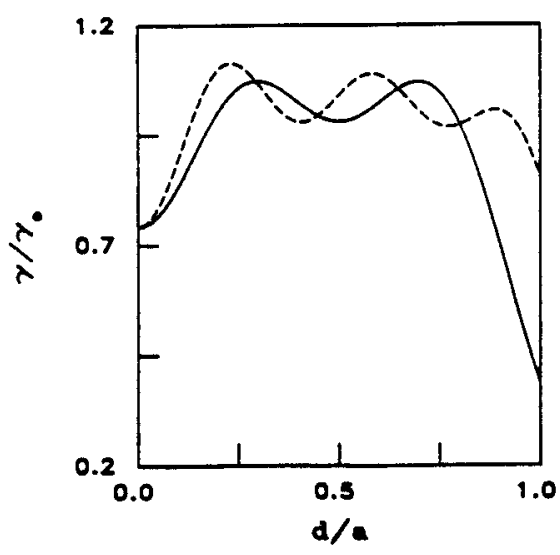

Fig. 5 - Samu as in Fig. 4, excopt that we have a glycerol particle instead of a cavity. (Ref. [15])

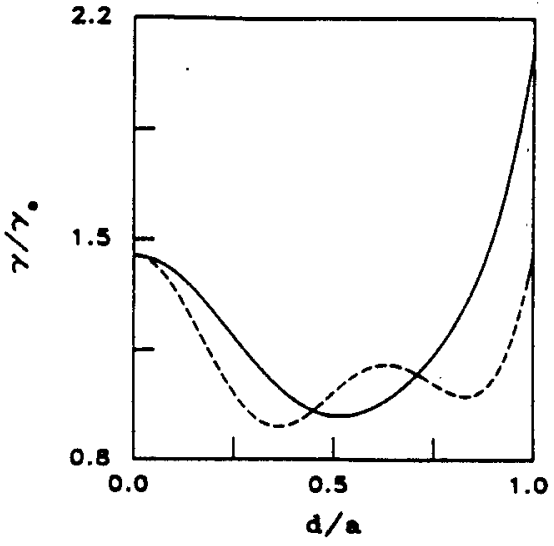

Fig. 4 - Same notations as in Fig. 3 but for a molecule inside a cavity. Only total rates are shown. (Ref. [15])

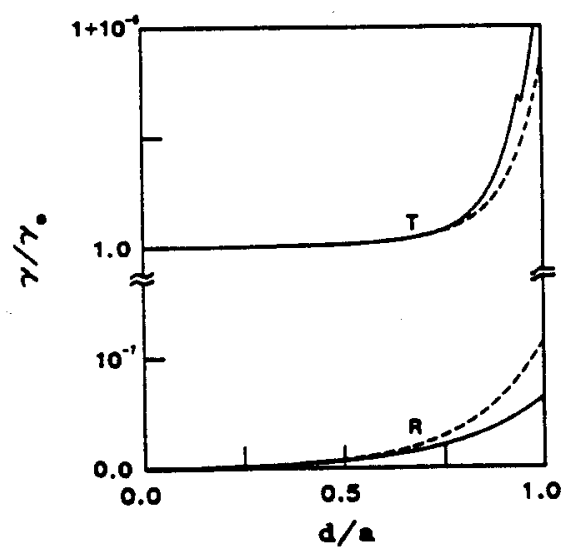

Ref. 6 - Same notations as in

Fig. 3 with the molecule embedded in a dissipative particle.

(Ref. [15]) 

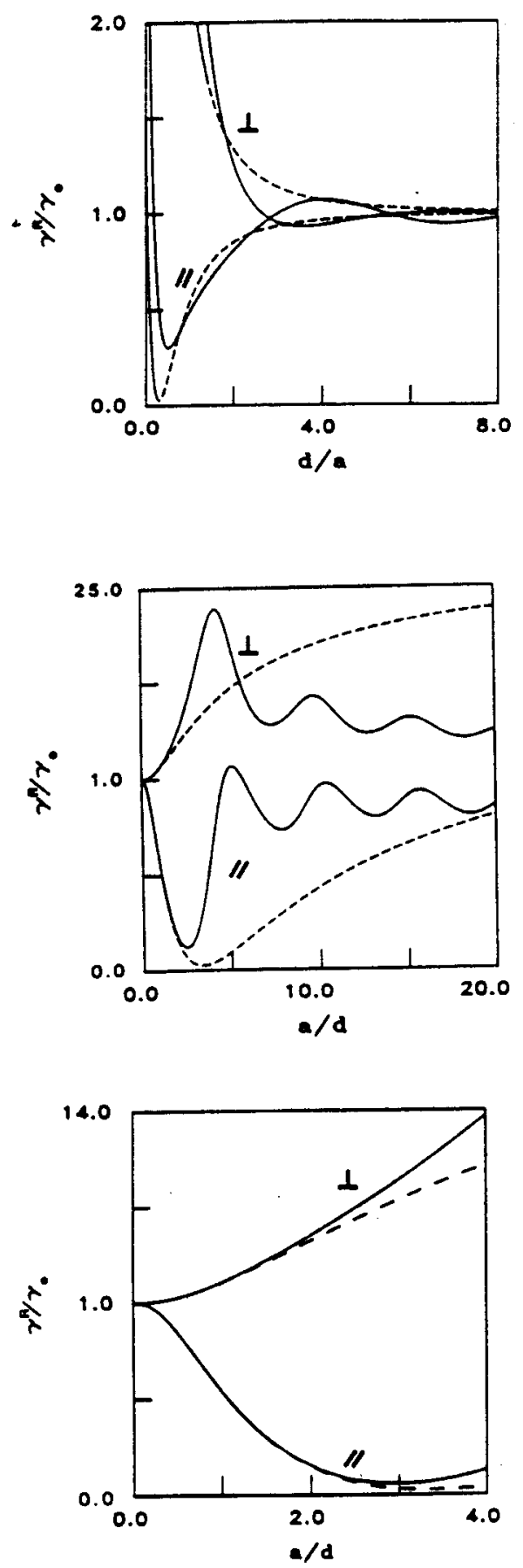

Fig. 7 - Comparison between the radiative transfer model in Ref. [35] (dashed curves) and the exact dynamical theory (solid curves for both radial $\left({ }^{+}\right)$and tangential (I)

molecular dipoles. The radius of the sphere is fixed at a $-400 \mathrm{~A}$. (Ref. [5I])

Fig. 8 - Same as in Fig. 7, but for the case with the molecule-surface distance fixed at d - 100 A. (Ref. [51])

Fig. 9 - Same as in Fig, 8, except that d is fixed at $50 \AA$. (Ref (5i)) 

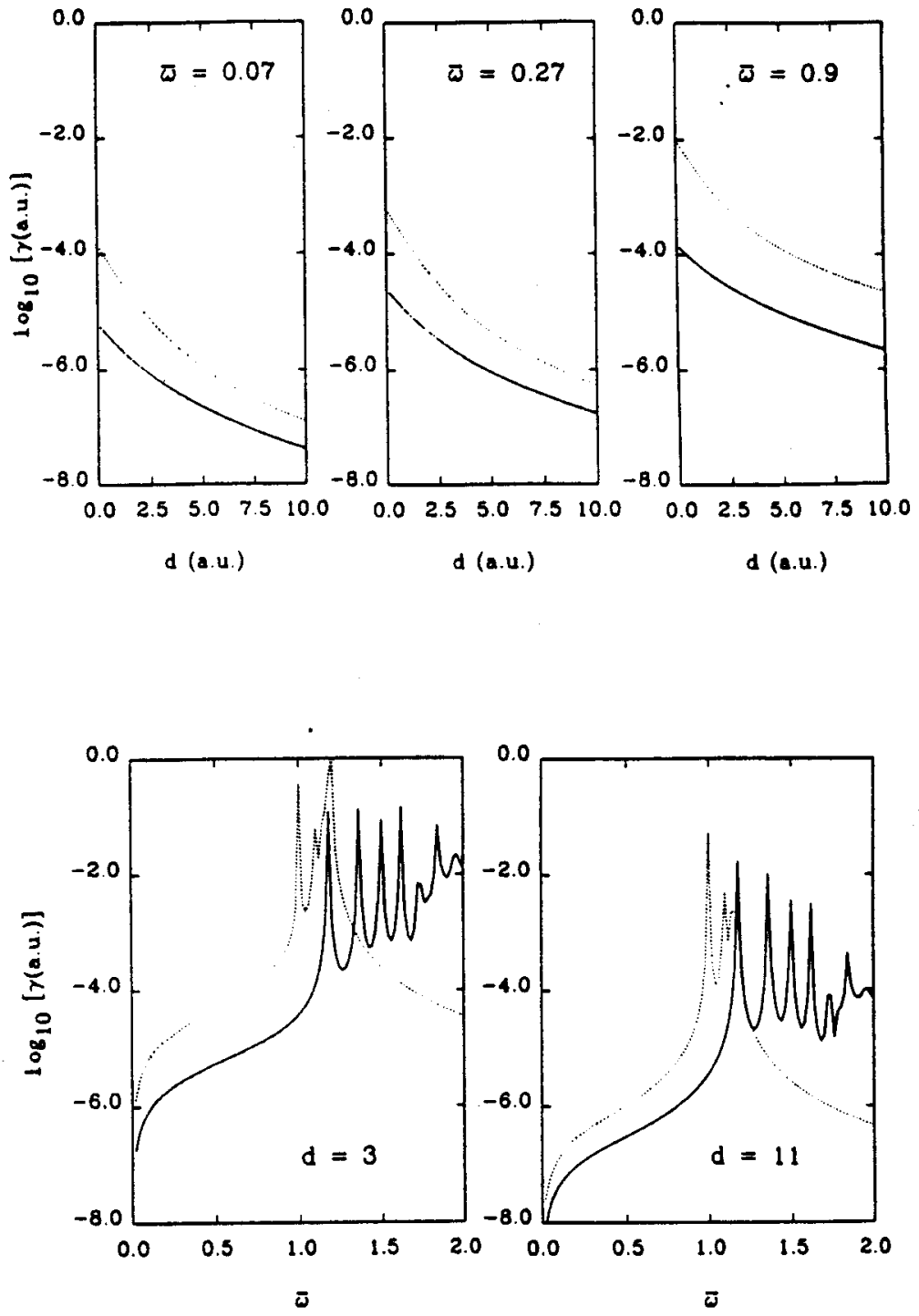

Fig. 10 - Illustrations of nonlocal effects (solid curves) on the decay rates for a radial molecular dipole outside a sodium sphere of radius a - 18.057 a.u. (Ref. [55]) The dotted lines ars locel results We show cases both for where the emission frequency is fixed and the distance is fixed, respectively. Note that atomic units are used in order to compare with Ref. [52]. 

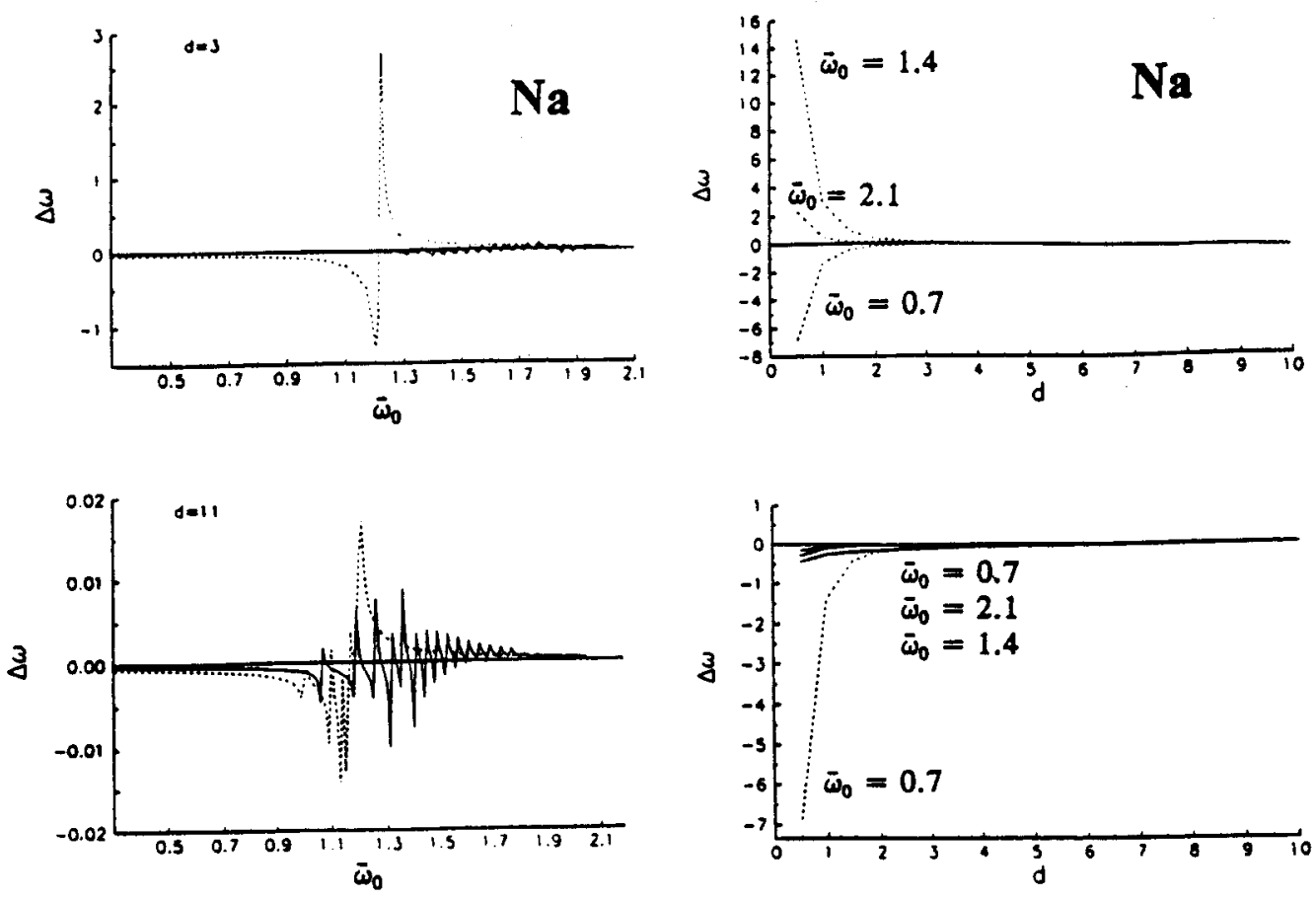

Fig. 11 - Same as in Fig. 10 bur the nonlocal effects are studied for the molecular frequency shifts with the sphere radius a -50.0 au. (Ref. [56]) 
$[55,56]$, and we are in a process of refining and further confirming our model by using more accurate microscopic expressions for $c(\vec{k}, \omega)$.

Conclusion

We have given above a brief survey of the very interesting and diversified topic of modified spectoscopic properties for fluorescing molecules close to a "microstructure." We have seen that, starting from Purcell some 50 years ago, this problem has interested researchers in different areas from nuclear to molecular spectroscopy, and for nontrivial environments ranging from a surface to a microparticle. There is relevance to both fundamental science and applied technology, such as fundamental QED and possible enhanced lasing action for embedded molecules, for example. We believe that there still exists a lot of future opportunities to uncover novel and interesting phenomenon in this study - both theoretical and experimental. For example, we are still awaiting for detailed experimental verification or rebuttal of the modeling results for dissipative particles as well as nonlocal effects at very close distances of the molecule from the surface boundary of the microstructure. In addition, experimental study of the effects from different particle geometries has yet to be done. It is relatively easy (though cumbersome) for the theoretical models to go beyond the spherical geometry for the particle [35], but it would be a greater challenge for experimentalists in this aspect. We hope that our very brief exploration of the subject will further stimulate more activities in this very rewarding area of spectroscopy.

\section{Acknowledgments}

PTL acknowledges the support of the Faculty Development Funds from Portland State University. This is Environmental Sciences and Resources Program Publication No. 297. TFG acknowledges support from NSF Grant CHE - 9196214 


\section{References}

[1] E. M. Purce11, Phys. Rev. 69, 681 (1946)

R. R. Chance, A. Prock and R. Silbey, Adv. Chem. Phys. 37, 1 (1978).

P. T. Leung and T. F. George, Spectroscopy 4, 35 (1989).

See e.g. D. Kleppner, Phys. Rev. Lett. 47, 233 (1981); A. J. Campillo, $J$. D. Eversole and H. B. Lin, Phys. Rev. Lete. 67, 437 (1991) and references therein.

(5) N. Liver, A. Nitzan, A. Amirav and J. Jortner, J. Chem. Phys. 88, 3516, (1988); A. Penner. A. Amirav, J. Jortner, A. Nitzan and J. Gersten, ibid.

(6) J. Martorell and N. M. Lawandy, Phys. Rev. Lett. 66,887 (1991)

[7a] E. Betzig and R. J. Chichester, Science 262, 1422 (1993) and other related articles in the same lssue.

(7b) X. S. Xle and R. C. Dunn, Science 265, 361 (1994); W. P. Ambrose, P. M. Goodwin, J. C. Martín and R. A. Keller, ibid., 364 (i994).

18] M. Fleischmann, P. J. Handra and A. J. McQuilian, Chem. Phys. Lett. 26, 163 (1974).

(9) M. Moskovits, Rev. Mod. Phys. 57, 783 (1985).

(10) G. M. Goncher, C. A. Parsons, C. B. Harris, J. Phys. Chem. 88, 4200 (i984); D. A. Jeiski, P. T. Leung and T. F. George, Int. Rev. Phys. Chem. 7. 179 (1988) and references therein.

(11) P. T. Leung and I. F. George, J. Chem. Phys. 85, 4729 (1986).

$12]$ J. Arias, P. K. Aravind and H. Metiu, Chem. Phys. Lett. 85, 404 (1982). P. T. Leung, 2. C. Wu, D. A. Jelsk1 and T. F. George, Phys. Rev. B. 36, 1475 (1987); P. T. Leung and T. F. George, ibid. 4664 (1987).

(14] N. Liver, A. Nitzan and K. F. Freed, J. Chem. Phys. 82, 3831 (1985).

Y. S. Kim, P. T. Leung and T. F. George, Surf. SCi. 195, 1 (1988).

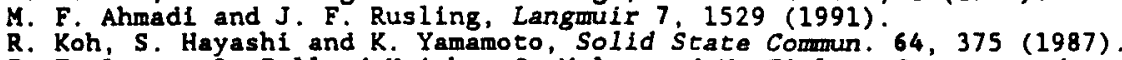
P. T. Leung, D. Pollard-Knight, G. Malan and M. Finlan, Sensors and . Actuators B (submitted)

[19] M. J. Pliska, E. D. Sanchez, P. T. Leung and T. F. George, Solid State Commun. 89, 397 (1994)

201 A. B. Myors and R. R. Birge, J. Chem. Phys, 73, 5314 (1980).

(21) S. Leutwyler, Chem. Phys. Lett. 107,284 (1984).

$22 \mathrm{~J}$. Gersten and A. Nitzan, J. Chem. Phys. 95, 686 (1991)

(23) See also, e.g. M. S. Kurdoglyan, Opt. Spectrosc. 75, 488 (1993) and references therein.

(24) See the first article quoted above in Ref. [5].

(25) D. Pines, D. Huppert and D. Avnir, J. Chem. Phys. 89, 1177 (1988).

[26] J S. Suh, M. Moskovits and J. Shakhesemampour, J. Phys.'Chem. 97, 1678 (i993).

[27] V. A. Markel, L. S. Muratov, M. I. Stockman and T. F. George, Phys. Rev. B 43. 8183 (1991).

[28] M. I. Stockman, V. M. Shalaev, M. Moskovits, R. Botet and T. F. George, Phys. Rev. B 46, 2821 (1992)

(29] D. A. Weitz and M. Oliveria, Phys. Rev. Lett. 52, 1433 (1984).

30] J. Martorell and N. M. Lawandy, Phys. Rev. Lett. 66, 887 (199i)

[31] N. M. Lawandy, R. M. Balachandran, A. S. L. Gomes and E. Sauvain, Nature 368,436 (1994).

[32] A. Sommerfeld, Ann. Phys. (Leipzig) 28, 665 (1909), ibid. 81, 1135 (1926). For review, see, e.g. R. R. Cavanagh, $E$. J. Heilweil and J. C. Stephenson, Surf. SCl. 299/300, 643 (1994). [34] See, H. A. Champton, A. R. Gallo, C. B. Harris, H. J. Robota and

[35] See, e.g., J. I. Gersten and A. Nitzan, Surf. Sci. 158, 165 (1985).

36) R. Ruppln, J. Chem. Phys. 76, 1681 (1982).

37) P. T. Leung and T. F. George, J. Chem. Phys. 87, 6722 (1987)

38) Y. S. KLn, P. T. Leung, T. F. George, Chem. Phy'. Lett. 152, 453 (1988).

39] H. Chew, J. Chem. Phys. 87. 1355 (1987).

$40)$ B. van dar Pol and H. Bremmer, Phil, Mag. 24, 141 (1937).

[41] V. A. Fock, Soviet Phys. JETP' 19 , 916 (1946) ' [n Russian]; see also v. A. Fock, Electromagnetic Diffraction and Propagation Problems (Perganon, New York, 965 ).

[42] M. Lewenstein, T. W. Mossberg and R. J. Glauber, Phys. Rev. Lett. 59, 775 (i987). 
(43] L. M. Folan, S. Arnold and S. D. Druger, Chem. Phys, Lett. 118, 322

[44] See e.g. A. S. Kwok and R. K. Chang, Opt. Lett. 17, 1262 (1992);

(44) lbid 18,1703 (ig93), and references therein.

[45] V. M. Agranovich and O. A. Dubovski1, Sov. Phys.-Solid State 12, 1631

[46] A. Zangw111, Physics at Surfaces (Cambridge University Press, Cambridge, 1988). P. 165 ff.

(47) R. Fuchs and F. Claro, Phys. Rev. B 35, 3722 (1987)

48 G. D. Mahan, Many-Particle Physics (Plenum, New York, 1981), pp. 420-447.

(49) G. Y. Hu and R. F. O-Connell, Phys. Rev. B 40, 3600 (1989).

[50] E. D. Palik, Ed., Handbook of Optical Constants of Sollds (Academic Press, New York, 1985).

(51) P. T. Leung, Y. S. KIm and T. F. George, J. Phys. Chem. 92, 6206 (1988).

(52] W. Ekardt and Z. Penzar, Phys. Rev. B 34,8444 (1986).

[53] K. D. Tsuel, E. W. Plummer, A. Liebsch, K. Kempa and P. Bakshi, Phys. Rev. Lett. 64, 44 (1990)

[54] F. Balzer, K. Bammel and H. G. Rubahn, J. Chem. Phys. 98, 7625 (1993).

[55] P. T. Leung, Phys. Rev. B 42, 7622 (1990).

[56] P. T. Leung and M. H. Hider, J. Chem. Phys. 98, 5019 (1993). 